\title{
Barbed expansion sphincter pharyngoplasty for the treatment of oropharyngeal collapse in obstructive sleep apnoea syndrome: A retrospective study on 17 patients
}

\section{1 | INTRODUCTION}

Expansion Sphincter Pharyngoplasty (ESP) is a promising surgical technique designed to treat patients with obstructive sleep apnoea syndrome (OSAS), characterised by oropharyngeal walls collapse, with reduced morbidity and high success rates compared to traditional uvulopalatopharyngoplasty (UPPP). ${ }^{1}$ In addition, the use of knotless barbed sutures has been recently described in OSAS oropharyngeal surgery to improve the biomechanical effect of sutures on tissue collapse with positive results. ${ }^{2,3}$

We present here our surgical technique for ESP using barbed suture, Barbed Expansion Sphincter Pharyngoplasty (BESP), and report the results observed in a group of 17 patients.

\section{2 | METHODS}

A retrospective evaluation of patients' clinical records was performed from December 2012 until January 2015.

\section{1 | Patient assessment}

Eligible patients had moderate-to-severe OSAS as assessed by a recent (within 6 months of screening) polysomnography (PSG), scored according to the American Academy of Sleep Medicine (AASM) 2007 and 2012 criteria, BMI $\leq 30 \mathrm{~kg} / \mathrm{m}^{2}$ without significant variations since the PSG, and either did not tolerate or refused therapy with continuous positive airway pressure (CPAP). For each patient demographic data, PSG date, BMI, AHI and ODI were retrieved, as well as the results of the Italian version of the ESS. ${ }^{4}$

\section{2 | Surgical planning}

Patients were selected for BESP based on the presence of oropharyngeal collapse, as determined by preoperative complete upper airways examination and drug-induced sleep endoscopy (DISE) using

Presented at the 1st EUROPEAN ADVANCED COURSE ON SURGICAL TECHNIQUES FOR SNORING AND OSAHS, organised by the University of Rome Tor Vergata and San Carlo Hospital in Rome, February 11th-12th, 2016, The Church Palace-Via Aurelia, 481 - 00165 Rome, Italy. the VOTE classification ${ }^{5}$ (Velar and Oropharyngeal) and did not present obstruction at other sites (base of Tongue and Epiglottis).

Patients complaining of nasal obstruction were scheduled to receive turbinate reduction surgery and/or septoplasty aimed at obtaining nasal symptom relief.

\section{3 | Surgical technique}

Surgery is performed under general anaesthesia, with the patient in the supine position and with orotracheal intubation. The oral cavity and oropharynx are exposed using a Boyle Davis mouth gag.

Bilateral tonsillectomy is performed, in patients who have already received tonsillectomy, and the oropharyngeal mucosa is removed to reveal the tonsillar pillars. A rectangular shaped strip of mucosa and the underlying submucosa are removed at the centre of the soft palate consisting of $0.5-0.7 \mathrm{~mm}$ in length and with width corresponding to the tonsillar fossae distance (Figure $1 \mathrm{~A}) .^{6} \mathrm{~A}$ single resorbable polydioxanone barbed bidirectional size 0 monofilament suture (Quill ${ }^{\mathrm{TM}}$ Suture Device-Surgical Specialties Corporation, MA, USA) is then used for BESP, the surgical steps are illustrated in Figures $1 \mathrm{~B}, 2 \mathrm{~A}, \mathrm{~B}$ and 3.

The edges of the tonsillar and palatal mucosal resection are secured together with single stitches using Vicryl 2-0 sutures.

\section{4 | Post-surgery assessment}

All patients were discharged with paracetamol $1 \mathrm{gr}$ three times a day before meals and chlorhexidine $0.2 \%$ mouth rinse after meals.

PSG was repeated postoperatively after 1 year. Duration of hospital stay and early complications, postoperative ESS, late complications and patient complaints were recorded at discharge from hospital and during follow-up visits. The last follow-up visit was performed after 1 year of surgery.

A successful procedure was defined as $50 \%$ reduction in $\mathrm{AHI}$ and $\mathrm{AHI}$ less than 20 events/hour, as described by Sher. ${ }^{7}$

\section{5 | Statistical analysis}

Statistical analysis of pre- and postoperative data was performed using the Shapiro-Wilk normality test and the Wilcoxon signed-rank 

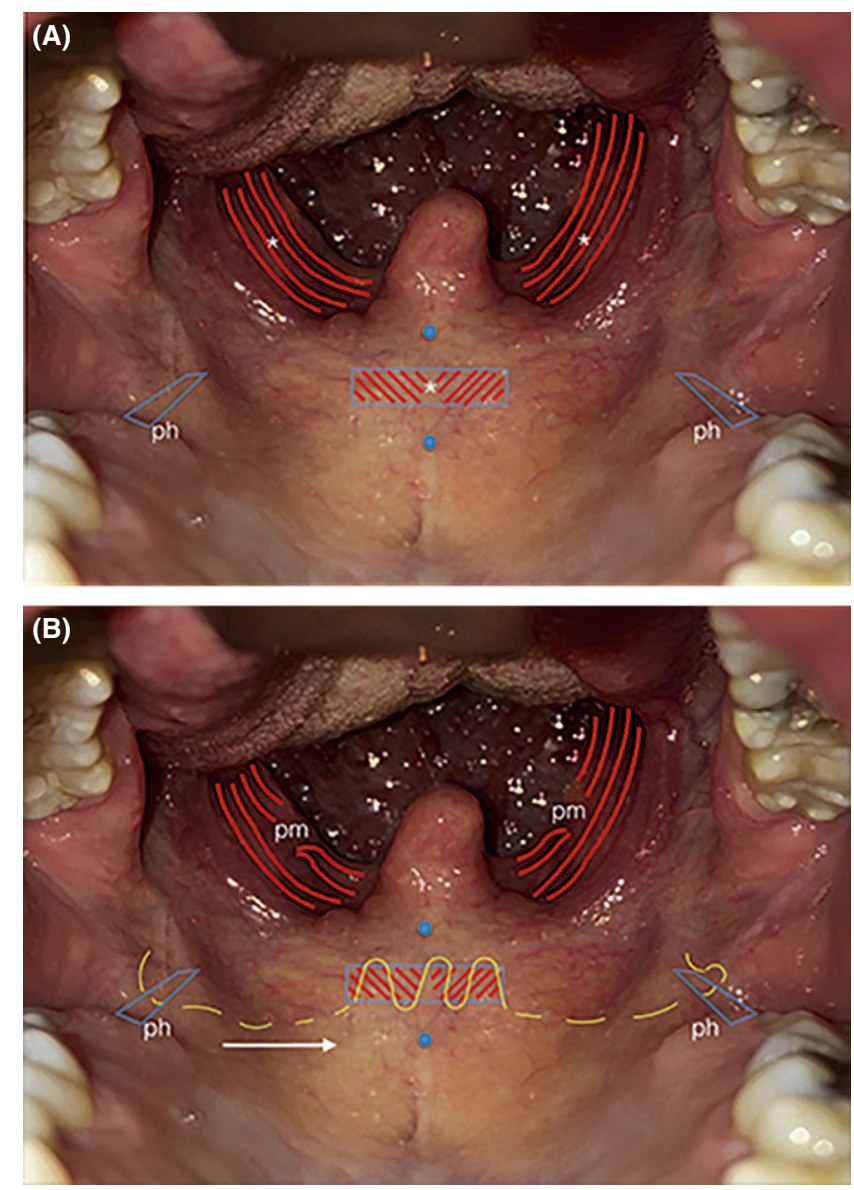

FIGURE 1 (A) Mucosal removal and exposure of palatoglossus, pharyngeal constrictor, palatopharyngeus and soft palate muscles $(*)$. Base of uvula (superior blue dot), posterior limit of hard palate (inferior blue dot) and pterygoid hamuli (ph) are also outlined. (B) Palatopharyngeus muscles (pm) are transected, and the suture thread (yellow line) is passed around the left pterygoid hamulus (ph) on one side and pulled out in the median demucosised portion of the soft palate. The barbed suture is then closed anteroposteriorly performing an anterior palatoplasty, and this step is completed passing the needle around the right pterygoid hamulus (ph). The arrow indicates the direction of the suture

test for matched samples. A 2-tailed $P$ value $<.05$ was considered significant.

\section{3 | RESULTS}

\section{1 | Patient data}

During the analysed period, 144 DISE were performed and 29 patients with Velar and Oropharyngeal collapse according to VOTE classification were identified. Seventeen eligible patients with $\mathrm{BMI} \leq$ $30 \mathrm{~kg} / \mathrm{m}^{2}$ who did not tolerate or refused CPAP (14 males and 3 females) underwent BESP. All patients met the inclusion criteria, and none were lost to follow-up. Three patients underwent septoplasty, 7 turbinate reduction surgery and one endoscopic polypectomy to relieve nasal obstruction.

\section{Keypoints}

- Expansion Sphincter Pharyngoplasty (ESP) was introduced in 2007 and represents a promising surgical technique to treat patients with oropharyngeal walls collapse with reduced morbidity and high success rates.

- Between December 2012 and January 2015, 17 patients underwent ESP at the department of Otorhinolaryngology of the University of Brescia (Italy), using barbed knotless sutures to improve the biomechanical effect of sutures on tissue collapse (BESP).

- Patients with moderate-to-severe OSAS and BMI < $30 \mathrm{~kg} / \mathrm{m}^{2}$, who did not tolerate or refused ventilation therapy, were selected for BESP based on the presence of oropharyngeal collapse, as determined by upper airway examination and drug-induced sleep endoscopy.

- Overall success of BESP was 94,1\%. Postoperative oxygen desaturation index (ODI) showed significant improvement $(P<.01)$. Likewise, Epworth Sleepiness Scale score was significantly reduced $(P<.05)$. No patient complained of uncontrolled pain after the procedure and/or reported unsatisfactory analgesia at followup visits.

- Albeit limited by the sample size and duration of followup, our experience confirms the validity of BESP in this selected patient population.

Patients' demographics, tonsil size, modified Mallampati score, Friedman score and preoperative PSG values are summarised in Table 1.

\subsection{Postoperative data}

Postoperative PSG data, ESS and BMI are listed in Table 1. Median postoperative BMI at 1 year was $28.5 \mathrm{~kg} / \mathrm{m}^{2}$ (IQR [29-26]). None of the patients presented significant weight variations. Median postoperative AHI was 7.8 events/hour (IQR [15.5-2.75]) and was significantly improved $(P<.01)$ compared to preoperative $A H I$ (Table 1). Overall success of BESP, as defined by Sher, was 94.1\%.

In addition, postoperative ODI showed statistically significant improvement ( $P<.01$; median, 11.2 events/hour; IQR [14-4.5]). Likewise, ESS was significantly reduced $(P<.05$; median, 4; IQR [6.5-2]).

In a single instance (patients number 11 in Table 1), the PSG outcome did not improve at the first control 1 year after surgery. Analysis of postoperative PSG in case 11 showed a mixed (mostly central) pattern of apnoeas, which was not apparent at preoperative PSG. The patient repeated DISE, which did not reveal significant upper airway obstruction, and was subsequently referred to a neurologist for further evaluation. 

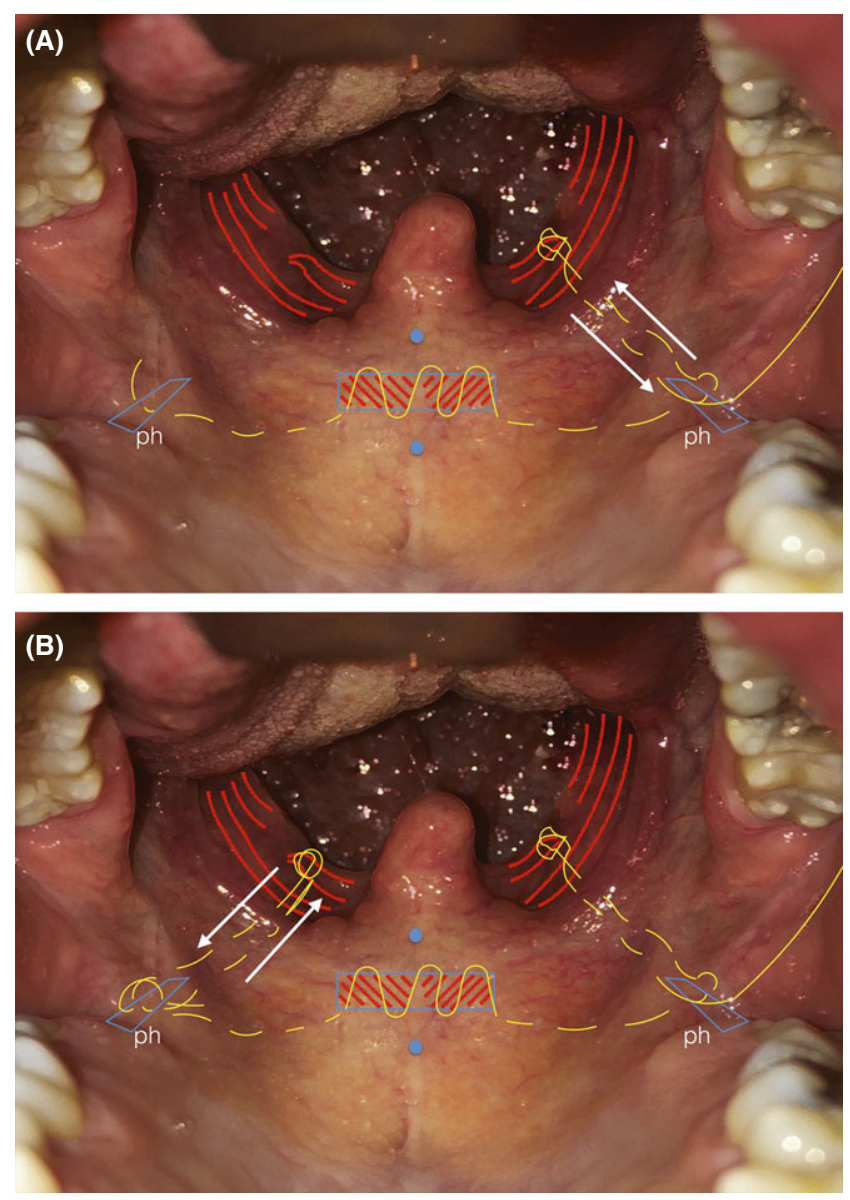

FIGURE 2 (A) The needle is reinserted in the level of the right pterygoid hamulus (ph) and pulled out of the right tonsillar fossa. Next, the transected palatopharyngeus muscle is hooked with the suture (yellow line) and pulled upwards and laterally, directing the needle towards the pterygomandibular raphe, which is twice submucosally surrounded with the suture. The arrows indicate the direction of the suture. (B) The same steps described in Figure 3 are performed on the right side in this figure. The arrows indicate the direction of the suture. Pterygoid hamulus (ph)

\section{3 | Postoperative course and complications}

No patient complained of uncontrolled pain after the procedure. Median hospital stay was 2 days (IQR [1-3]).

Complications related to BESP included one chipped tooth caused by mouth gag displacement, 1 case of tonsillar haemorrhage, 1 case of acute postoperative infection and 1 case of temporary episodic nasal liquid regurgitation, which regressed spontaneously after 2 weeks. No patient reported postoperative alterations in speech or taste after 1 year. On average, patients started eating soft foods on the first postoperative day and normal diet was resumed after 15-20 days. One patient experienced wound dehiscence after sneezing, which prolonged the mucosal healing, and normal diet was resumed after 30 days.

\section{DISCUSSION}

Our technique is a modified version of ESP using barbed sutures. With BESP, we achieved a success rate in line with other ESP case

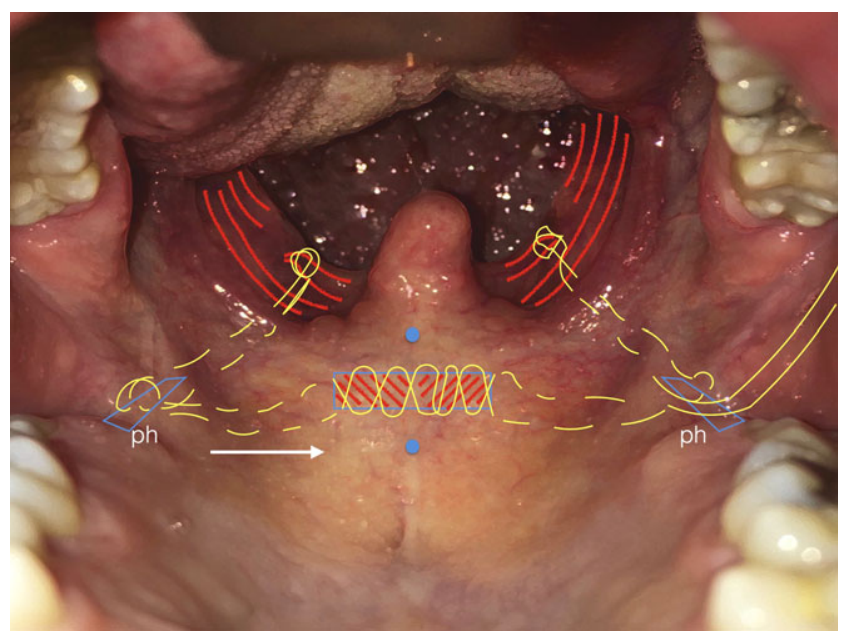

FIGURE 3 The needle on the right side is brought close to the other by repeating the anterior palatoplasty suture (second layer), and the two threads are tied together on the left side. The arrow indicates the direction of the suture. Pterygoid hamulus (ph)

series. Albeit limited, our experience confirms the validity of the ESP approach in patients selected with DISE, and overall success of BESP and associated treatments, as defined by Sher, was $94.1 \%$.

BESP success was not limited to $\mathrm{AHI}$, but also other important PSG values and subjective measures were positively affected. In particular, ODI showed a statistically significant improvement and offers an appraisal of surgical result in terms of reduction in cardiovascular disease risk. ${ }^{8}$

Concerning subjective outcome measures, ESS was significantly improved after BESP and, importantly, all patients preoperatively complaining of significant somnolence (ESS >10) resolved their symptoms.

Eleven patients underwent nasal surgery together with BESP to relieve symptomatic nasal obstruction. A recent meta-analysis indicates that nasal surgery has a slightly significant impact on $\mathrm{AHI}$, with a weighted mean $\mathrm{AHI}$ reduction of 4.15 . $^{9}$ Still, even taking into account the possible role of nasal surgery, much of the AHI reduction that we observed (median difference between pre- and postoperative $\mathrm{AHI}$ : 17.4) can be reasonably attributed to BESP.

Most importantly, only four of the patients had to resort to treatment with CPAP after surgery. As low adherence or objection to CPAP treatment was the main reasons why patients underwent surgery, it is evident that BESP had a favourable impact on the quality of life of the majority of patients, sparing them from an unwanted or bothersome therapy. ${ }^{10}$

BESP is associated with low morbidity and minimal complications and appears to be well tolerated by patients. Postoperative discomfort is also low, and patients can resume their normal activities in a reasonably short time.

In our series, postoperative PSG showed that BESP was not successful in one case. Indeed, central apnoeas, which were not evident during preoperative PSG, played a key role in the persistence of sleep apnoea. It is possible that, in this case, the hypoxic respiratory drive was reduced due to chronic OSAS-related hypoxia. 
TAB LE 1 Patients 'demographics and pre-postoperative polysomnography (PSG) values, patient number (nr), Epworth Sleepiness Scale (ESS), Body Mass Index (BMI), Apnea/Hypopnea Index (AHI), tonsil size (TS), Modified Mallampati Scale (MMP), Friedman Anatomic Scale (FAS),

Oxygen Desaturation Index (ODI) and interquartile range (IQR)

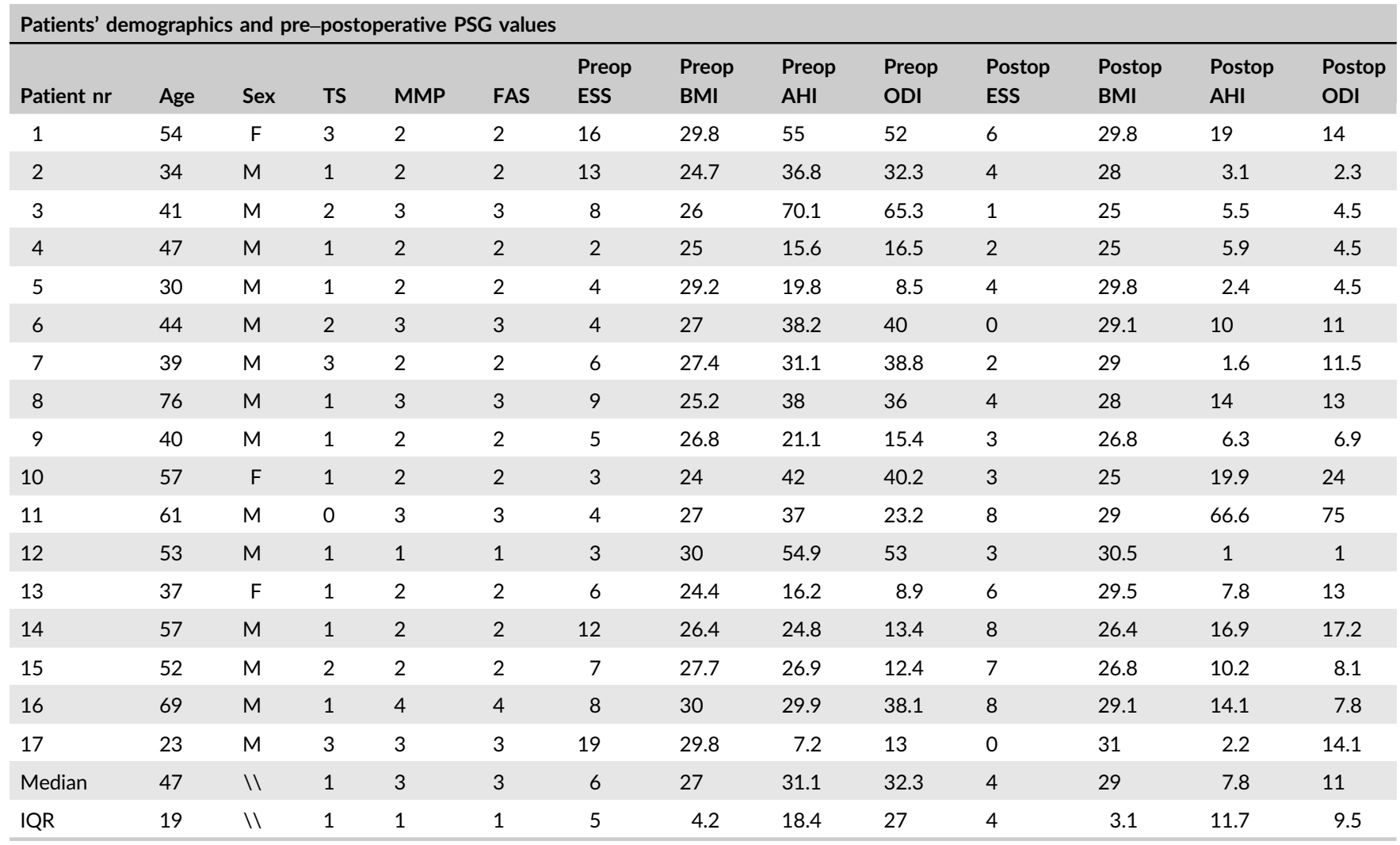

\section{5 | CONCLUSION}

In conclusion, our data on BESP confirm the validity of the technique L. Pianta on selected patients with moderate-to-severe OSAS, BMI $<30 \mathrm{~kg} / \mathrm{m}^{2}$ G. Bertazzoni and Velar and Oropharyngeal collapse according to VOTE classification assessed with clinical examination and DISE. BESP respects the anatomy of the pharynx, resection of soft tissues is limited, and the rate of complications is very low. In our experience, the use of barbed suture increased the ease of execution and stability of pharyngoplasty.

Our data on BESP add evidence on the efficacy of ESP, which will probably play an increasing role in OSAS surgery in selected patients with oropharyngeal collapse, as a stand-alone procedure or as part of a multilevel approach in patients with multisite collapse.

R. Morello iD

P. Perotti

P. Nicolai

Department of Otorhinolaryngology - Head and Neck Surgery, University of Brescia,

Brescia, Italy

Correspondence

L. Pianta, Departmemt of Otorhinolaryngology, University of Brescia,

Brescia, Italy.

Email: lucapianta@virgilio.it

\section{CONFLICT OF INTEREST}

None to declare.

\section{ETHICAL CONSIDERATION}

No ethics committee requirements were needed for this article.

\section{ORCID}

R. Morello iD http://orcid.org/0000-0003-4460-997X

\section{REFERENCES}

1. Pang KP, Woodson BT. Expansion sphincter pharyngoplasty: a new technique for the treatment of obstructive sleep apnea. Otolaryngol Head Neck Surg. 2007;137:110-114

2. Vicini C, Hendawy E, Campanini A, et al. Barbed reposition pharyngoplasty (BRP) for OSAHS: a feasibility, safety, efficacy and teachability pilot study. \&quot;We are on the giant's shoulders\&quot. Eur Arch Otorhinolaryngol. 2015;272:3065-3070.

3. Mantovani M, Rinaldi V, Torretta S, Carioli D, Salamanca F, Pignataro L. Barbed Roman blinds technique for the treatment of obstructive 
sleep apnea: how we do it? Eur Arch Otorhinolaryngol. 2016;273:517-523.

4. Vignatelli L, Plazzi G, Barbato A, et al. Italian version of the Epworth sleepiness scale: external validity. Neurol Sci. 2003;23:295-300.

5. Kezirian EJ, Hohenhorst W, de Vries N. Drug-induced sleep endoscopy: the VOTE classification. Eur Arch Otorhinolaryngol. 2011;268:1233-1236.

6. Salamanca F, Costantini F, Mantovani M, et al. Barbed anterior pharyngoplasty: an evolution of anterior palatoplasty. Acta Otorhinolaryngol Ital. 2014;34:434-438. http://www.ncbi.nlm.nih.gov/ pubmed/25762837. Accessed November 9, 2016.

7. Sher AE, Schechtman KB \& Piccirillo JF. The efficacy of surgical modifications of the upper airway in adults with obstructive sleep apnea syndrome. Sleep. 1996;19:156-177. http://www.ncbi.nlm.nih. gov/pubmed/8855039. Accessed October 3, 2014.

8. Seif $\mathrm{F}$, Patel SR, Walia $\mathrm{H}$, et al. Association between obstructive sleep apnea severity and endothelial dysfunction in an increased background of cardiovascular burden. J Sleep Res. 2013;22:443-451.

9. Wu J, Zhao G, Li Y, et al. Apnea-hypopnea index decreased significantly after nasal surgery for obstructive sleep apnea: a meta-analysis. Medicine. 2017;96:e6008.

10. Richard W, Venker J, den Herder C, et al. Acceptance and long-term compliance of nCPAP in obstructive sleep apnea. Eur Arch Otorhinolaryngol. 2007;264:1081-1086. 